\title{
On optimal investment with processes of long or negative memory
}

\author{
Huy N. Chau* Miklós Rásonyi*
}

March 28, 2017

\begin{abstract}
We consider the problem of utility maximization for investors with power utility functions. Building on the earlier work Larsen et al. (2016), we prove that the value of the problem is a Fréchet-differentiable function of the drift of the price process, provided that this drift lies in a suitable Banach space.

We then study optimal investment problems with non-Markovian driving processes. In such models there is no hope to get a formula for the achievable maximal utility. Applying results of the first part of the paper we provide first order expansions for certain problems involving fractional Brownian motion either in the drift or in the volatility. We also point out how asymptotic results can be derived for models with strong mean reversion.
\end{abstract}

\section{Introduction}

Portfolio optimization is one of the most important questions in mathematical finance, however, explicit solutions are obtained occasionally in some concrete and simple market models. As a tool for solving optimization problems, the dynamic programming principle shows its power in the Markovian realm. Nevertheless, the non-Markovian world exhibits significant difficulties which require different approaches.

Recent literature on optimal investment has paid considerable attention to approximate solutions. More precisely, in many cases where no closed-form result can be found, series expansions could be provided for both the value function and the parameters of the optimal strategy, see e.g. Gerhold et al. (2014, 2012, 2013); Guasoni and Weber (2015). In other words, those difficult optimization problems were considered as a perturbation of some well-understood benchmark in an appropriate manner. In the present article we wish to take a similar, "perturbation" view on optimal investment in certain classes of non-Markovian market models.

Our starting point is the main result in Larsen et al. (2016). In an incomplete market, where the stock price dynamics are given by a continuous

\footnotetext{
* Alfréd Rényi Institute of Mathematics, Hungarian Academy of Sciences, Budapest. The authors were supported by the "Lendület" grant LP2015-6 of the Hungarian Academy of Sciences. We thank Gordan Žitković for helpful discussions related to this paper.
} 
semimartingale, these authors prove that a power investor's value function is Gâteaux differentiable with respect to the market price of risk. We push this idea further by proving that the value function also has a Fréchet derivative on suitable domains. Since the space of market prices of risk is infinite dimensional, the discrepancy between these two concepts of differentiability is significant, see Remark 3.5 below.

We then apply this new result to some particular market models exhibiting long memory or antipersistence (negative memory). Our examples will be driven by a fractional Brownian motion (fBm) whose memory features will be characterized by its Hurst parameter $H \in(0,1)$. It is well-known that fBms have long memory for $H>1 / 2$ and negative memory for $H<1 / 2$, see Chapter 3 of Giraitis et al. (2012) for a detailed discussion of these concepts, especially Definitions 3.1.2 and 3.1.3.

One of the principal messages of our paper is that the value function of a power utility maximizer is differentiable with respect to the Hurst parameter, with an explicitly given derivative. This helps to deepen our knowledge on the effect of memory in optimization. Some further applications will also be mentioned.

The paper is organized as follows. In Section 2, we introduce the market model and recall the pertinent terminology. In Section 3 , we extend the result of Larsen et al. (2016) by proving that the value function is also Fréchet differentiable. Section 4 introduces some financial markets driven by fractional Brownian motion. Section 5 treats models with strongly mean-reverting drifts. Section 6 comments on possible future work. Section 7 provides some necessary computations with fBms.

\section{The model}

Let $(\Omega, \mathcal{F}, \mathbb{P})$ be a probability space, and $\mathbb{F}:=\left(\mathcal{F}_{t}\right)_{t \in[0, T]}$ be a filtration satisfying the usual conditions. Let $\mathbb{L}^{0}$ denote the set of (a.s. equivalence classes of) scalar random variables, endowed with the topology of convergence in probability. The symbol $\mathbb{L}^{p}$ refers to the usual Banach space of $p$-integrable random variables.

For a continuous $\mathbb{F}$-local martingale $M$, we denote

$\mathcal{P}_{M}^{2}:=\left\{\left(\pi_{t}\right)_{t \in[0, T]}: \pi\right.$ is $\mathbb{F}-$ progressively measurable, $\int_{0}^{T} \pi_{t}^{2} d\langle M\rangle_{t}<\infty, \mathbb{P}-$ a.s. $\}$,

where $\langle M\rangle$ denotes the quadratic variation of the local martingale $M$. For each $\lambda \in \mathcal{P}_{M}^{2}$, we define a continuous semimartingale $S^{\lambda}=\mathcal{E}\left(R^{\lambda}\right)$ where

$$
R_{t}^{\lambda}=\int_{0}^{t} \lambda_{s} d\langle M\rangle_{s}+M_{t}
$$

and $\mathcal{E}$ denotes the stochastic exponential. The process $R^{\lambda}$ is interpreted as the return of the process $S^{\lambda}$ and the process $\lambda$ is called the market price of risk in the literature. The financial market consists of the risky asset $S^{\lambda}$ and a zerointerest bond. Given $x>0$, we denote by $\mathcal{X}^{\lambda}(x)$ the set of all nonnegative 
wealth processes starting from initial capital $x$, i.e.

$$
\mathcal{X}^{\lambda}(x):=\left\{x \mathcal{E}\left(\int_{0} \pi_{t} d R_{t}^{\lambda}\right): \pi \in \mathcal{P}_{M}^{2}\right\} .
$$

The optimization problem. The investor's preferences are modeled by a power utility function

$$
U(x):=\frac{x^{p}}{p}, \quad x>0,
$$

where $p<0$ is the risk-aversion parameter. For $x>0$, we consider the optimal investment problem

$$
u^{\lambda}(x):=\max _{X \in \mathcal{X}^{\lambda}(x)} \mathbb{E} U\left(X_{T}\right) .
$$

The dual optimization problem. For $\lambda \in \mathcal{P}$, the stochastic exponential $Z^{\lambda}$ given by

$$
Z_{t}^{\lambda}:=\mathcal{E}(-\lambda \cdot M)_{t}, \quad t \in[0, T],
$$

is a strictly positive local martingale. This process figures prominently in the study of the dual domain, which is described now. For $y>0$, we define

$$
\begin{gathered}
\mathcal{Y}^{\lambda}(y):=\left\{Y>0: Y \text { is a càdlàg supermartingale such that } Y_{0}=y\right. \text { and } \\
\left.Y X \text { is a supermartingale for every } X \in \mathcal{X}^{\lambda}(1)\right\} .
\end{gathered}
$$

The conjugate function $V:(0, \infty) \rightarrow \mathbb{R}$ is defined by

$$
V(y):=\sup _{x>0}(U(x)-x y)=\frac{y^{-q}}{q}, \quad \text { where } q:=\frac{p}{1-p} \in(-1,0) .
$$

The dual value function is defined as follows

$$
v^{\lambda}(y):=\inf _{Y \in \mathcal{Y}^{\lambda}(y)} \mathbb{E}\left[V\left(Y_{T}\right)\right], \quad y>0 .
$$

It is shown in Corollary 3.3 of Larsen and Žitković (2007) that if $v^{\lambda}(y)$ is finite then the minimizer $\widehat{Y}^{\lambda}$ for the dual problem always has the form

$$
\widehat{Y}^{\lambda}=Z^{\lambda} \widehat{H}^{\lambda}, \quad \text { for some } \widehat{H} \in \mathcal{H},
$$

where $\mathcal{H}$ denotes the set of all positive local martingales $H$ with $H_{0}=1$ that are strongly orthogonal to $M$.

Remark 2.1 (No arbitrage conditions). The requirement that $\lambda \in \mathcal{P}_{M}^{2}$ is not enough for the validity of the NFLVR condition, see Delbaen and Schachermaver (2006), since a candidate for a local martingale density can easily be a strict local martingale. However, this is the minimal condition needed to ensure that the optimization problem is well-posed. Indeed, the process $Z^{\lambda}$ plays the role of a supermartingale deflator, in the terminology of Karatzas and Kardaras (2007), and its presence implies that the condition NUPBR of that paper holds true. For recent duality results under the condition NUPBR, we refer to Chau et al. (2015). 


\section{Fréchet differentiability}

Larsen et al. (2016) prove that the value function $u^{\lambda}$ is Gâteaux differentiable with respect to $\lambda$ on a certain, suitable domain. We will show that even a Fréchet derivative can be found in a smaller domain, which requires stronger regularity of the market price of risk.

Let $\alpha>1$ be a real number such that $(-q) \alpha<1$ and let $\beta>1$ be the Hölder conjugate of $\alpha$, i.e. $\frac{1}{\alpha}+\frac{1}{\beta}=1$. In short, let $\beta>1$ be such that $\frac{-q \beta}{\beta-1}<1$. For such $\beta$ we define

$$
\|\lambda\|_{\beta}:=\mathbb{E}\left[\left(\int_{0}^{T}\left(\lambda_{t}\right)^{2} d\langle M\rangle_{t}\right)^{\beta}\right]^{\frac{1}{2 \beta}},
$$

and set $\mathcal{D}_{\beta}:=\left\{\lambda \in \mathcal{P}_{M}^{2}:\|\lambda\|_{\beta}<+\infty\right\}$. It is easily checked that $\mathcal{D}_{\beta}$ is a Banach space with the given norm.

Following Definition 2.7 of Larsen and Žitković (2007), a metrizable topology $\tau$ on some subset $\mathcal{A} \subset \mathcal{P}_{M}^{2}$ is said to be appropriate if the mapping $\lambda \mapsto Z_{T}^{\lambda}$ from $\mathcal{A}$ into $\mathbb{L}_{+}^{0}$ is continuous, when $\mathcal{A}$ is endowed with $\tau$, and $\mathbb{L}^{0}$ with the topology of convergence in probability.

Lemma 3.1. The norm given in (4) induces an appropriate topology on $\mathcal{D}_{\beta}$.

Proof. Suppose that $\lambda^{n} \rightarrow \lambda$ in $\mathcal{D}_{\beta}$. Hölder's inequality shows that

$$
\mathbb{E}\left[\int_{0}^{T}\left|\lambda_{t}^{n}-\lambda_{t}\right|^{2} d\langle M\rangle_{t}\right] \leq \mathbb{E}\left[\left(\int_{0}^{T}\left|\lambda_{t}^{n}-\lambda_{t}\right|^{2} d\langle M\rangle_{t}\right)^{\beta}\right]^{1 / \beta} \rightarrow 0 .
$$

Since the norm $\mathbb{E}\left[\int_{0}^{T} \lambda_{t}^{2} d\langle M\rangle_{t}\right]^{\frac{1}{2}}$ induces an appropriate topology by Proposition A.1 of Larsen and Žitković (2007), it holds that $Z_{T}^{\lambda^{n}} \rightarrow Z_{T}^{\lambda}$ in probability. Therefore, the norm (4) also induces an appropriate topology.

Now, we recall a result of Larsen and Žitković (2007).

Theorem 3.2. For any $\lambda \in \mathcal{D}_{\beta}$, the function $u^{\lambda}:(0, \infty) \rightarrow \mathbb{R}$ is finite-valued. The mapping $(\lambda, x) \rightarrow u^{\lambda}(x)$ is jointly continuous.

Proof. This follows from Theorem 2.12 of Larsen and Žitkovic (2007) noting that the set $\mathcal{D}_{\beta}$ is trivially $V$-relatively compact in the present case (see Definition 2.10 of Larsen and Žitkovic (2007)) and the topology induced by (4) is appropriate, see Lemma 3.1 above.

For simplicity, we also write $u^{\lambda}:=u^{\lambda}(1)$ and $v^{\lambda}:=v^{\lambda}(1)$. Furthermore, Larsen et al. (2016) prove that the value function is not only continuous but also Gâteaux differentiable with respect to the market price of risk. We recall their Theorem 3.1.

Theorem 3.3 (The Gâteaux derivative). Let $\lambda, \lambda^{\prime} \in \mathcal{P}_{M}^{2}$ such that

$$
\int_{0}^{T}\left(\lambda_{t}^{\prime}\right)^{2} d\langle M\rangle_{t} \in \mathbb{L}^{1-p} \quad \text { and } \quad \int_{0}^{T} \lambda_{t}^{\prime} d R_{t}^{\lambda} \in \cup_{s>1-p} \mathbb{L}^{s} .
$$


Then we have

$$
\begin{aligned}
& \left.\frac{d}{d \varepsilon} u^{\lambda+\varepsilon \lambda^{\prime}}\right|_{\varepsilon=0}:=\lim _{\varepsilon \rightarrow 0} \frac{1}{\varepsilon}\left(u^{\lambda+\varepsilon \lambda^{\prime}}-u^{\lambda}\right)=p u^{\lambda} \mathbb{E}\left[\frac{d \tilde{\mathbb{P}}^{\lambda}}{d \mathbb{P}} \int_{0}^{T} \lambda_{t}^{\prime} d R_{t}^{\lambda}\right], \\
& \left.\frac{d}{d \varepsilon} v^{\lambda+\varepsilon \lambda^{\prime}}\right|_{\varepsilon=0}:=\lim _{\varepsilon \rightarrow 0} \frac{1}{\varepsilon}\left(v^{\lambda+\varepsilon \lambda^{\prime}}-v^{\lambda}\right)=q v^{\lambda} \mathbb{E}\left[\frac{d \tilde{\mathbb{P}}^{\lambda}}{d \mathbb{P}} \int_{0}^{T} \lambda_{t}^{\prime} d R_{t}^{\lambda}\right],
\end{aligned}
$$

where the probability measure $\tilde{\mathbb{P}}^{\lambda}$ is defined by $\frac{d \tilde{\mathbb{P}}^{\lambda}}{d \mathbb{P}}=\frac{1}{u^{\lambda}} U\left(\widehat{X}_{T}^{\lambda}\right)=\frac{1}{v^{\lambda}} V\left(\widehat{Y}_{T}^{\lambda}\right)$, and $\widehat{X}_{T}^{\lambda}, \widehat{Y}_{T}^{\lambda}$ are the solutions of (1) and (3), respectively.

By the choice of $\beta$, we see that $1-p<\beta$, therefore any $\lambda^{\prime} \in \mathcal{D}_{\beta}$ satisfies also the integrability conditions (5) in Theorem 3.3. Now, we push the idea of Larsen et al. (2016) further by proving that the value function is also Fréchet differentiable.

Theorem 3.4 (The Fréchet derivative). Let $\frac{-q \beta}{\beta-1}<1$ hold. Then the mapping $\lambda \mapsto u^{\lambda}(x)$ from $\mathcal{D}_{\beta}$ to $\mathbb{R}$ is Fréchet differentiable.

Proof. Using Larsen et al. (2016), the Gâteaux derivative of $u^{\lambda}$ at $\lambda$ in the direction $\lambda^{\prime}$ (see Theorem 3.3) can be rewritten as

$$
p \frac{u^{\lambda}}{v^{\lambda}} \mathbb{E}\left[V\left(\widehat{Y}_{T}^{\lambda}\right) \int_{0}^{T} \lambda_{t}^{\prime} d R_{t}^{\lambda}\right] .
$$

First, we prove that the Gâteaux derivative, as a linear operator acting on $\lambda^{\prime} \in \mathcal{D}_{\beta}$ is continuous, at every point $\lambda \in \mathcal{D}_{\beta}$. Indeed, the linearity of ([6) with respect to $\lambda^{\prime}$ is obvious and thus we only need to consider its continuity on $\mathcal{D}_{\beta}$. Let $\lambda^{\prime \prime} \rightarrow \lambda^{\prime}$ in $\mathcal{D}_{\beta}$. Using the Hölder inequality and the Burkholder-DavisGundy inequality, we estimate

$$
\begin{aligned}
& \mathbb{E}\left[V\left(\widehat{Y}_{T}^{\lambda}\right) \int_{0}^{T}\left|\lambda_{t}^{\prime}-\lambda_{t}^{\prime \prime}\right| d R_{t}^{\lambda}\right] \leq \mathbb{E}\left[V\left(\widehat{Y}_{T}^{\lambda}\right)^{\alpha}\right]^{\frac{1}{\alpha}} \mathbb{E}\left[\left|\int_{0}^{T}\right| \lambda_{t}^{\prime}-\lambda_{t}^{\prime \prime}\left|\lambda_{t} d\langle M\rangle_{t}\right|^{2 \beta}\right]^{\frac{1}{2 \beta}} \\
& \left.\left.\left.+\mathbb{E}\left[V\left(\widehat{Y}_{T}^{\lambda}\right)^{\alpha}\right]^{\frac{1}{\alpha}} \mathbb{E}\left[\left|\int_{0}^{T}\right| \lambda_{t}^{\prime}-\lambda_{t}^{\prime \prime}\left|d M_{t}\right|^{2 \beta}\right]^{\frac{1}{2 \beta}}\right]^{\beta}\right]\left.^{\frac{1}{2 \beta}}[\mid]^{T}\left|\lambda_{t}\right|^{2} d\langle M\rangle_{t}\right|^{\beta}\right]^{\frac{1}{2 \beta}} \\
& \leq \mathbb{E}\left[V\left(\widehat{Y}_{T}^{\lambda}\right)^{\alpha}\right]^{\frac{1}{\alpha}} \mathbb{E}\left[\left|\int_{0}^{T}\right| \lambda_{t}^{\prime}-\left.\lambda_{t}^{\prime \prime}\right|^{2} d\langle M\rangle_{t}\right. \\
& +\mathbb{E}\left[V\left(\widehat{Y}_{T}^{\lambda}\right)^{\alpha}\right]^{\frac{1}{2 \beta}} \mathbb{E}\left[\left|\int_{0}^{T}\right| \lambda_{t}^{\prime}-\left.\left.\lambda_{t}^{\prime \prime}\right|^{2} d\langle M\rangle_{t}\right|^{\frac{1}{\alpha}},\right.
\end{aligned}
$$

which tends to zero and the first claim is proved. 
Now it suffices to prove that the Gâteaux derivative is a continuous function of $\lambda$, see Proposition 3.2.15 of Drábek and Milota (2013), i.e. we need to prove that the quantity in (6) is continuous on $\mathcal{D}_{\beta}$ (in the operator norm topology).

Letting $\tilde{\lambda} \rightarrow 0$ in $\mathcal{D}_{\beta}$, we compute

$$
\begin{aligned}
\frac{u^{\lambda+\tilde{\lambda}}}{v^{\lambda+\tilde{\lambda}}} \mathbb{E}\left[V\left(\widehat{Y}_{T}^{\lambda+\tilde{\lambda}}\right) \int_{0}^{T} \lambda_{t}^{\prime} d R_{t}^{\lambda+\tilde{\lambda}}\right]-\frac{u^{\lambda}}{v^{\lambda}} \mathbb{E}\left[V\left(\widehat{Y}_{T}^{\lambda}\right) \int_{0}^{T} \lambda_{t}^{\prime} d R_{t}^{\lambda}\right] \\
=\mathbb{E}\left[\frac{u^{\lambda+\tilde{\lambda}}}{v^{\lambda+\tilde{\lambda}}} V\left(\widehat{Y}_{T}^{\lambda+\tilde{\lambda}}\right) \int_{0}^{T} \lambda_{t}^{\prime} d M_{t}-\frac{u^{\lambda}}{v^{\lambda}} V\left(\widehat{Y}_{T}^{\lambda}\right) \int_{0}^{T} \lambda_{t}^{\prime} d M_{t}\right] \\
+\mathbb{E}\left[\frac{u^{\lambda+\tilde{\lambda}}}{v^{\lambda+\tilde{\lambda}}} V\left(\widehat{Y}_{T}^{\lambda+\tilde{\lambda}}\right) \int_{0}^{T} \lambda_{t}^{\prime}\left(\lambda_{t}+\tilde{\lambda}_{t}\right) d\langle M\rangle_{t}-\frac{u^{\lambda}}{v^{\lambda}} V\left(\widehat{Y}_{T}^{\lambda}\right) \int_{0}^{T} \lambda_{t}^{\prime} \lambda_{t} d\langle M\rangle_{t}\right] .
\end{aligned}
$$

Using Hölder's inequality, the first term in the RHS of (7) is bounded by

$$
\begin{aligned}
& \mathbb{E}\left[\left|\left(\frac{u^{\lambda+\tilde{\lambda}}}{v^{\lambda+\tilde{\lambda}}} V\left(\widehat{Y}_{T}^{\lambda+\tilde{\lambda}}\right)-\frac{u^{\lambda}}{v^{\lambda}} V\left(\widehat{Y}_{T}^{\lambda}\right)\right) \int_{0}^{T} \lambda_{t}^{\prime} d M_{t}\right|\right] \\
& \leq \mathbb{E}\left[\left|\frac{u^{\lambda+\tilde{\lambda}}}{v^{\lambda+\tilde{\lambda}}} V\left(\widehat{Y}_{T}^{\lambda+\tilde{\lambda}}\right)-\frac{u^{\lambda}}{v^{\lambda}} V\left(\widehat{Y}_{T}^{\lambda}\right)\right|^{\alpha}\right]^{\frac{1}{\alpha}} \mathbb{E}\left[\left|\int_{0}^{T} \lambda_{t}^{\prime} d M_{t}\right|^{2 \beta}\right]^{\frac{1}{2 \beta}} \\
& \leq C \mathbb{E}\left[\left|\frac{u^{\lambda+\tilde{\lambda}}}{v^{\lambda+\tilde{\lambda}}} V\left(\widehat{Y}_{T}^{\lambda+\tilde{\lambda}}\right)-\frac{u^{\lambda}}{v^{\lambda}} V\left(\widehat{Y}_{T}^{\lambda}\right)\right|^{\alpha}\right]^{\frac{1}{\alpha}}\left[\left(\int_{0}^{T}\left(\lambda_{t}^{\prime}\right)^{2} d\langle M\rangle_{t}\right)^{\beta}\right]^{\frac{1}{2 \beta}},
\end{aligned}
$$

where the last bound is due to the Burkholder-Davis-Gundy inequality. By Theorem 3.2 we have that $u^{\lambda+\tilde{\lambda}} \rightarrow u^{\lambda}$ as $\tilde{\lambda} \rightarrow 0$ in $\mathcal{D}_{\beta}$. In addition, $V\left(\widehat{Y}_{T}^{\lambda+\tilde{\lambda}}\right) \rightarrow$ $V\left(\widehat{Y}_{T}^{\lambda}\right)$ in probability, see Lemma 3.5 of Larsen and Žitković (2007). This implies

$$
\frac{u^{\lambda+\tilde{\lambda}}}{v^{\lambda+\tilde{\lambda}}} V\left(\widehat{Y}_{T}^{\lambda+\tilde{\lambda}}\right)-\frac{u^{\lambda}}{v^{\lambda}} V\left(\widehat{Y}_{T}^{\lambda}\right) \rightarrow 0, \quad \text { in probability as } \tilde{\lambda} \rightarrow 0
$$

Furthermore, the inequality $|x+y|^{\alpha} \leq 2^{\alpha-1}\left(|x|^{\alpha}+\left|y^{\alpha}\right|\right.$ ) (with $\alpha>1$ ) yields

$$
\left|\frac{u^{\lambda+\tilde{\lambda}}}{v^{\lambda+\tilde{\lambda}}} V\left(\widehat{Y}_{T}^{\lambda+\tilde{\lambda}}\right)-\frac{u^{\lambda}}{v^{\lambda}} V\left(\widehat{Y}_{T}^{\lambda}\right)\right|^{\alpha} \leq 2^{\alpha-1}\left(\left(\frac{u^{\lambda+\tilde{\lambda}}}{v^{\lambda+\tilde{\lambda}}}\right)^{\alpha}\left(\widehat{Y}_{T}^{\lambda+\tilde{\lambda}}\right)^{-q \alpha}+\left(\frac{u^{\lambda}}{v^{\lambda}}\right)^{\alpha}\left(\widehat{Y}_{T}^{\lambda}\right)^{-q \alpha}\right) .
$$

It is noticed that $(-q) \alpha<1$, and $\sup _{\tilde{\lambda}} \mathbb{E}\left[\widehat{Y}_{T}^{\lambda+\tilde{\lambda}}\right] \leq 1$, the theorem of de la ValléePoussin implies that the family $\left(\widehat{Y}_{T}^{\lambda+\tilde{\lambda}}\right)^{-q \alpha}$ is uniformly integrable. Together with (8), these imply

$$
\mathbb{E}\left[\left|\frac{u^{\lambda+\tilde{\lambda}}}{v^{\lambda+\tilde{\lambda}}} V\left(\widehat{Y}_{T}^{\lambda+\tilde{\lambda}}\right)-\frac{u^{\lambda}}{v^{\lambda}} V\left(\widehat{Y}_{T}^{\lambda}\right)\right|^{\alpha}\right]^{\frac{1}{\alpha}} \rightarrow 0 .
$$


The second term on the RHS of (7) is estimated as follows,

$$
\begin{aligned}
& \mathbb{E}\left[\left|\frac{u^{\lambda+\tilde{\lambda}}}{v^{\lambda+\tilde{\lambda}}} V\left(\widehat{Y}_{T}^{\lambda+\tilde{\lambda}}\right) \int_{0}^{T} \lambda_{t}^{\prime}\left(\lambda_{t}+\tilde{\lambda}_{t}\right) d\langle M\rangle_{t}-\frac{u^{\lambda}}{v^{\lambda}} V\left(\widehat{Y}_{T}^{\lambda}\right) \int_{0}^{T} \lambda_{t}^{\prime} \lambda_{t} d\langle M\rangle_{t}\right|\right] \\
\leq & \mathbb{E}\left[\left|\frac{u^{\lambda+\tilde{\lambda}}}{v^{\lambda+\tilde{\lambda}}} V\left(\widehat{Y}_{T}^{\lambda+\tilde{\lambda}}\right) \int_{0}^{T} \lambda_{t}^{\prime}\left(\lambda_{t}+\tilde{\lambda}_{t}\right) d\langle M\rangle_{t}-\frac{u^{\lambda+\tilde{\lambda}}}{v^{\lambda+\tilde{\lambda}}} V\left(\widehat{Y}_{T}^{\lambda+\tilde{\lambda}}\right) \int_{0}^{T} \lambda_{t}^{\prime} \lambda_{t} d\langle M\rangle_{t}\right|\right] \\
+ & \mathbb{E}\left[\left|\frac{u^{\lambda+\tilde{\lambda}}}{v^{\lambda+\tilde{\lambda}}} V\left(\widehat{Y}_{T}^{\lambda+\tilde{\lambda}}\right) \int_{0}^{T} \lambda_{t}^{\prime} \lambda_{t} d\langle M\rangle_{t}-\frac{u^{\lambda}}{v^{\lambda}} V\left(\widehat{Y}_{T}^{\lambda}\right) \int_{0}^{T} \lambda_{t}^{\prime} \lambda_{t} d\langle M\rangle_{t}\right|\right] .
\end{aligned}
$$

By using the generalized Hölder inequality in the same way as above, we obtain the following estimations

$$
\begin{aligned}
& \mathbb{E}\left[\left|\frac{u^{\lambda+\tilde{\lambda}}}{v^{\lambda+\tilde{\lambda}}} V\left(\widehat{Y}_{T}^{\lambda+\tilde{\lambda}}\right) \int_{0}^{T} \lambda_{t}^{\prime} \lambda_{t} d\langle M\rangle_{t}-\frac{u^{\lambda}}{v^{\lambda}} V\left(\widehat{Y}_{T}^{\lambda}\right) \int_{0}^{T} \lambda_{t}^{\prime} \lambda_{t} d\langle M\rangle_{t}\right|\right] \leq \\
& \mathbb{E}\left[\left|\frac{u^{\lambda+\tilde{\lambda}}}{v^{\lambda+\tilde{\lambda}}} V\left(\widehat{Y}_{T}^{\lambda+\tilde{\lambda}}\right)-\frac{u^{\lambda}}{v^{\lambda}} V\left(\widehat{Y}_{T}^{\lambda}\right)\right|^{\alpha}\right]^{\frac{1}{\alpha}} \mathbb{E}\left[\left(\int_{0}^{T} \lambda_{t}^{2} d\langle M\rangle_{t}\right)^{\beta}\right]^{\frac{1}{2 \beta}} \mathbb{E}\left[\left(\int_{0}^{T}\left(\lambda_{t}^{\prime}\right)^{2} d\langle M\rangle_{t}\right)^{\beta}\right]^{\frac{1}{2 \beta}}
\end{aligned}
$$

and

$$
\begin{aligned}
& \mathbb{E}\left[\left|\frac{u^{\lambda+\tilde{\lambda}}}{v^{\lambda+\tilde{\lambda}}} V\left(\widehat{Y}_{T}^{\lambda+\tilde{\lambda}}\right) \int_{0}^{T} \lambda_{t}^{\prime}\left(\lambda_{t}+\tilde{\lambda}_{t}\right) d\langle M\rangle_{t}-\frac{u^{\lambda+\tilde{\lambda}}}{v^{\lambda+\tilde{\lambda}}} V\left(\widehat{Y}_{T}^{\lambda+\tilde{\lambda}}\right) \int_{0}^{T} \lambda_{t}^{\prime} \lambda_{t} d\langle M\rangle_{t}\right|\right] \leq \\
& \mathbb{E}\left[\left|\frac{u^{\lambda+\tilde{\lambda}}}{v^{\lambda+\tilde{\lambda}}} V\left(\widehat{Y}_{T}^{\lambda+\tilde{\lambda}}\right)\right|^{\alpha}\right]^{\frac{1}{\alpha}} \mathbb{E}\left[\left(\int_{0}^{T} \tilde{\lambda}_{t}^{2} d\langle M\rangle_{t}\right)^{\beta}\right]^{\frac{1}{2 \beta}} \mathbb{E}\left[\left(\int_{0}^{T}\left(\lambda_{t}^{\prime}\right)^{2} d\langle M\rangle_{t}\right)^{\beta}\right] .
\end{aligned}
$$

From these estimations and (6), we conclude that the Gâteaux derivative is continuous and hence the proof is complete.

Remark 3.5. We wish to point out that Theorem 3.4 is not a mere technical improvement with respect to previous results (differentiability in a stronger sense is established) but it opens the door to essentially new developments that were hitherto inaccessible.

We briefly explain a general scheme here that can be implemented in various models. Sections 4 and 5 below carry out this scheme in concrete models, but the main idea seems to be applicable in great generality.

Let $\Theta \subset \mathbb{R}^{m}$ be an open set and consider a parametric class of market models of the type as in Section 2 i.e. let $v: \Theta \rightarrow \mathcal{D}_{\beta}$ be a mapping. The market model corresponding to parameter $\theta \in \Theta$ will have drift $v(\theta)$.

Assume that $v$ is differentiable. Then the composition of $v$ with the mapping $\lambda \rightarrow u^{\lambda}, \lambda \in \mathcal{D}_{\beta}$ is also differentiable by Theorem 3.4 (note that for this conclusion to hold, Gâteaux differentiability does not suffice). It follows that we can find a first order expansion of the mapping $\theta \rightarrow u^{v(\theta)}$ in $\theta$, i.e. the 
sensitivity of the optimal expected utility with respect to the model parameter can be studied. We stress that it is Theorem 3.4 that makes such an approach feasible: Gâteaux differentiability allows the study of linear perturbations for the drift while Fréchet differentiability permits non-linear perturbations as well.

Remark 3.6. Fix $\lambda$. Denoting by $\pi^{\lambda}$ the optimal strategy corresponding to drift $\lambda$, one expects that $\pi^{\lambda}$ is nearly optimal for the problem with drift $\lambda^{\prime}$ as well, provided that the latter is "close enough" to $\lambda$.

When we fix a direction $\lambda^{\prime}$ and we are only interested in approximating $\pi^{\lambda+\varepsilon \lambda^{\prime}}$ then this issue has been thoroughly studied in Subsection 3.2 of Larsen et al. (2016). They obtain, under suitable technical conditions, that $\pi^{\lambda}$ is $O\left(\varepsilon^{2}\right)$ optimal for the problem with drift $\lambda+\varepsilon \lambda^{\prime}$. Moreover, in a Brownian setting (see Larsen et al. (2016) for details), a correction term can be found that improves on this:

$$
\pi^{\lambda}+\varepsilon \frac{\lambda^{\prime}+p \gamma^{B}}{1-p}
$$

is an $O\left(\varepsilon^{3}\right)$-optimal control for the drift $\lambda+\varepsilon \lambda^{\prime}$. Here $\gamma^{B}$ is a process coming from the martingale representation theorem applied to $\int_{0}^{T} \pi_{t}^{\lambda} \lambda_{t}^{\prime} d\langle M\rangle_{t}$ under a certain probability $\tilde{\mathbb{P}}^{\lambda}$ (see Theorem 3.3) that is constructed from the solution of the utility maximization problem at $\lambda$.

As seen from the above, the correction term is not simple because $\gamma^{B}$ can be found only in very specific cases. The sufficient conditions for these results to hold are difficult to check in concrete models due to the presence of $\tilde{\mathbb{P}}^{\lambda}$ on which one has little grasp.

Nonetheless we quickly sketch a (rather trivial and rather stringent) sufficient condition that ensures $O(\varepsilon)$ optimality of $\pi^{\lambda}$ for $\lambda^{\prime}$ with $\left\|\lambda^{\prime}-\lambda\right\|_{\beta}<\varepsilon$, irrespective of the direction of $\lambda^{\prime}-\lambda$. For some more general comments on related problems, see Section [6. Let $\lambda, \lambda^{\prime} \in \mathcal{D}_{\beta}$.

One can write

$$
\begin{array}{r}
\left|U\left(\mathcal{E}\left(\int_{0}^{T} \pi_{t}^{\lambda} d R_{t}^{\lambda}\right)\right)-U\left(\mathcal{E}\left(\int_{0}^{T} \pi_{t}^{\lambda} d R_{t}^{\lambda^{\prime}}\right)\right)\right|= \\
\frac{1}{|p|} \exp \left\{p \int_{0}^{T} \pi_{t}^{\lambda} d M_{t}+p \int_{0}^{T} \pi_{t}^{\lambda} \lambda_{t} d\langle M\rangle_{t}-\frac{p}{2} \int_{0}^{T}\left(\pi_{t}^{\lambda}\right)^{2} d\langle M\rangle_{t}\right\} \times \\
\left|\exp \left\{p \int_{0}^{T} \pi_{t}^{\lambda}\left(\lambda_{t}^{\prime}-\lambda_{t}\right) d\langle M\rangle_{t}\right\}-1\right|
\end{array}
$$

so, using $\left|e^{x}-1\right| \leq|x| \sup _{|\varepsilon| \leq 1} e^{\varepsilon|x|}$ and the Cauchy inequality,

$$
\begin{array}{r}
\mathbb{E}\left|U\left(\mathcal{E}\left(\int_{0}^{T} \pi_{t}^{\lambda} d R_{t}^{\lambda}\right)\right)-U\left(\mathcal{E}\left(\int_{0}^{T} \pi_{t}^{\lambda} d R_{t}^{\lambda^{\prime}}\right)\right)\right| \leq \\
\frac{1}{|p|} \mathbb{E}^{1 / 2} \exp \left\{2 p \int_{0}^{T} \pi_{t}^{\lambda} d M_{t}+2 p \int_{0}^{T} \pi_{t}^{\lambda} \lambda_{t} d\langle M\rangle_{t}-p \int_{0}^{T}\left(\pi_{t}^{\lambda}\right)^{2} d\langle M\rangle_{t}\right\} \\
|p| \mathbb{E}^{1 / 2}\left[\left(\int_{0}^{T}\left|\pi_{t}^{\lambda}\right|\left|\lambda_{t}^{\prime}-\lambda_{t}\right| d\langle M\rangle_{t}\right)^{2} \sup _{|\varepsilon| \leq 1} \exp \left\{2 \varepsilon p \int_{0}^{T}\left|\pi_{t}^{\lambda}\right|\left|\lambda_{t}^{\prime}-\lambda_{t}\right| d\langle M\rangle_{t}\right\}\right] .
\end{array}
$$


Note that

$$
\begin{array}{r}
\left|u^{\lambda^{\prime}}-\mathbb{E} U\left(\mathcal{E}\left(\int_{0}^{T} \pi_{t}^{\lambda} d R_{t}^{\lambda^{\prime}}\right)\right)\right| \leq \\
\left|u^{\lambda^{\prime}}-u^{\lambda}\right|+\mathbb{E}\left|U\left(\mathcal{E}\left(\int_{0}^{T} \pi_{t}^{\lambda} d R_{t}^{\lambda}\right)\right)-U\left(\mathcal{E}\left(\int_{0}^{T} \pi_{t}^{\lambda} d R_{t}^{\lambda^{\prime}}\right)\right)\right| .
\end{array}
$$

Here the first term is $\leq C_{1}\left\|\lambda-\lambda^{\prime}\right\|_{\beta}$ with some constant $C_{1}>0$ by Theorem 3.4. If

$$
C_{2}:=\mathbb{E} \exp \left\{2 p \int_{0}^{T} \pi_{t}^{\lambda} d M_{t}+2 p \int_{0}^{T} \pi_{t}^{\lambda} \lambda_{t} d\langle M\rangle_{t}-p \int_{0}^{T}\left(\pi_{t}^{\lambda}\right)^{2} d\langle M\rangle_{t}\right\}
$$

is finite (which is a condition similar in spirit to those of Larsen et al. (2016)) and $\exp \left\{2 p \int_{0}^{T}\left|\pi_{t}^{\lambda}\right|\left|\lambda_{t}^{\prime}-\lambda_{t}\right| d\langle M\rangle_{t}\right\}<K$, for some constant $K$ then the second term is dominated by

$$
C_{2} K \mathbb{E}^{1 / 2}\left(\int_{0}^{T}\left|\pi_{t}^{\lambda}\right|\left|\lambda_{t}^{\prime}-\lambda_{t}\right| d\langle M\rangle_{t}\right)^{2}
$$

and the latter expression is expected to be small if $\lambda-\lambda^{\prime}$ is small in an appropriate sense, depending on the concrete class of models and on what we know about the optimizer $\pi^{\lambda}$. For instance, if $\pi^{\lambda}, \lambda^{\prime}, \lambda$ were bounded and

$$
l d t \leq d\langle M\rangle_{t} \leq L d t
$$

held for some constants $l, L>0$ then there would be such a $K$ and (10) would be dominated by constant times $\left\|\lambda-\lambda^{\prime}\right\|_{\beta}$, we omit further details.

\section{Optimization with fBM}

We recall here the definition of fractional Brownian motion and some related properties.

Definition 4.1. The standard fractional Brownian motion $\left(B_{t}^{H}\right)_{t \in \mathbb{R}}$ with Hurst parameter $0<H<1$ is defined as a continuous centered Gaussian process with

$$
\operatorname{Cov}\left(B_{t}^{H}, B_{s}^{H}\right)=\frac{1}{2}\left(|t|^{2 H}+|s|^{2 H}-|t-s|^{2 H}\right), \quad t, s \in \mathbb{R} .
$$

For $H>\frac{1}{2}$, the increments of $B^{H}$ are positively correlated; for $H<\frac{1}{2}$, they are negatively correlated. Furthermore, for every $\kappa \in(0, H)$, its sample paths are almost surely Hölder continuous with index $\kappa$. It is worth noting that for $H>\frac{1}{2}$, the process exhibits long-range dependence. In the following, we will often use the integral representation of $B^{H}$,

$$
B_{t}^{H}=\int_{\mathbb{R}} K^{H}(t, s) d W_{s}, \quad H \in\left(0, \frac{1}{2}\right) \cup\left(\frac{1}{2}, 1\right),
$$


where

$$
K^{H}(t, s):=C(H)\left((t-s)_{+}^{H-\frac{1}{2}}-(-s)_{+}^{H-\frac{1}{2}}\right), t \geq 0, s \in \mathbb{R},
$$

and $W$ is a Brownian motion. Here we adopt the usual convention $x_{+}:=$ $\max (0, x) . C(H)$ is the normalizing constant and defined by

$$
C(H):=\left(\int_{0}^{\infty}\left((1+s)^{H-\frac{1}{2}}-s^{H-\frac{1}{2}}\right)^{2} d s+\frac{1}{2 H}\right)^{-\frac{1}{2}}=\frac{(2 H \sin \pi H \Gamma(2 H))^{\frac{1}{2}}}{\Gamma\left(H+\frac{1}{2}\right)} .
$$

The following notations will be used in the sequel to simplify our computations.

$$
\begin{aligned}
& C_{1}(\alpha):=\int_{0}^{\infty}\left((1+s)^{\alpha}-(s)^{\alpha}\right)^{2} d s+\int_{0}^{1}(1-s)^{2 \alpha} d s, \\
& C_{2}(\alpha):=\int_{0}^{\infty}\left((1+s)^{\alpha} \ln (1+s)-(s)^{\alpha} \ln s\right)^{2} d s+\int_{0}^{1}\left((1-s)^{\alpha} \ln (1-s)\right)^{2} d s .
\end{aligned}
$$

If $\alpha \in\left(-\frac{1}{2}, \frac{1}{2}\right)$, the functions $C_{1}(\alpha)$ and $C_{2}(\alpha)$ are well-defined.

The use of fractional Brownian motion in stock price modeling has a long history. Most importantly, fBms may provide an explanation for the presence of the long-range dependence phenomenon in stock return, as suggested by many empirical studies, for example Willinger et al. (1999). In a seminal work, Comte and Renault (1998) proposed to model log-volatility by an fBm with Hurst parameter $H>\frac{1}{2}$ in order to capture the long memory property. By contrast, Fukasawa (2011) and Gatheral et al. (2014) suggest that the choice $H<\frac{1}{2}$ enables us to be consistent with observed term structure of the volatility skew.

In this paper, we discuss the effect of the Hurst parameter on the value function rather than the choice of this parameter. In Subsection 4.1, the market model is chosen such that only the drift exhibits long or negative memory. In Subsection 4.2, the log-volatility takes memory into account.

\subsection{Model 1}

We assume that the market price of risk evolves as a fractional OrnsteinUhlenbeck process satisfying $d \lambda_{t}^{H}=-\alpha \lambda_{t}^{H} d t+d B_{t}^{H}$, where $\alpha>0$ and $B^{H}$ is a fractional Brownian motion with parameter $H \in(0,1)$. This equation admits an explicit solution,

$$
\lambda_{t}^{H}=e^{-\alpha t}\left(\lambda_{0}^{H}+\int_{0}^{t} e^{\alpha u} d B_{u}^{H}\right) .
$$

Here, the stochastic integral with respect to fBM is simply a pathwise Riemann Stieltjes integral, see Proposition A.1 of Cheridito et al. (2003). Assume that the price of a risky asset follows $S^{H}=\mathcal{E}\left(R^{H}\right)$, where

$$
d R_{t}^{H}=\lambda_{t}^{H} d t+\rho d W_{t}+\sqrt{1-\rho^{2}} d B_{t},
$$

$\rho \in(0,1)$ and $B$ is a Brownian motion independent of $W$. Let $\mathbb{F}$ be the completed natural filtration of $(W, B)$. 
Proposition 4.2. The mapping

$$
\begin{aligned}
(0,1) & \rightarrow \mathcal{D}_{\beta} \\
H & \mapsto\left(\lambda_{t}^{H}\right)_{t \in[0, T]}
\end{aligned}
$$

is Fréchet differentiable and its derivative is the process

$$
D \lambda_{t}^{H}:=\int_{\mathbb{R}} \partial_{H} K^{H}(t, s) d W_{s}-\alpha e^{-\alpha t} \int_{0}^{t}\left(\int_{\mathbb{R}} \partial_{H} K^{H}(u, s) d W_{s}\right) e^{\alpha u} d u .
$$

Proof. First, since $\lambda_{t}^{H}$ is a Gaussian random variable, we easily check that $\lambda^{H} \in$ $\mathcal{D}_{\beta}$. It suffices to prove that

$$
\frac{1}{|\varepsilon|}\left\|\lambda^{H+\varepsilon}-\lambda^{H}-\varepsilon D \lambda^{H}\right\|_{\beta} \rightarrow 0 \quad \text { as } \varepsilon \rightarrow 0 .
$$

Using Hölder's inequality, we have

$$
\left\|\lambda^{H+\varepsilon}-\lambda^{H}-\varepsilon D \lambda^{H}\right\|_{\beta}^{2 \beta} \leq c \int_{0}^{T} \mathbb{E}\left[\left|\lambda_{t}^{H+\varepsilon}-\lambda_{t}^{H}-\varepsilon D \lambda_{t}^{H}\right|^{2 \beta}\right] d t .
$$

In what follows, the constant $c$ may vary from line to line. We compute $\int_{0}^{t} e^{\alpha u} d B_{u}^{H}=e^{\alpha t} B_{t}^{H}-e^{\alpha t} B_{0}^{H}-\alpha \int_{0}^{t} B_{u}^{H} e^{\alpha u} d u$, by integrating by parts. Therefore, we obtain

$$
\begin{aligned}
\lambda_{t}^{H+\varepsilon}-\lambda_{t}^{H}-\varepsilon \partial_{H} \lambda_{t}^{H}=\underbrace{B_{t}^{H+\varepsilon}-B_{t}^{H}-\varepsilon \int_{\mathbb{R}} \partial_{H} K^{H}(t, s) d W_{s}}_{I_{\varepsilon}^{1}} \\
-\alpha \underbrace{\alpha \int_{0}^{t} e^{-\alpha(t-u)}\left(B_{u}^{H+\varepsilon}-B_{u}^{H}-\varepsilon \int_{\mathbb{R}} \partial_{H} K^{H}(u, s) d W_{s}\right) d u}_{I_{\varepsilon}^{2}} .
\end{aligned}
$$

It is immediate that

$$
\varepsilon^{-2 \beta}\left\|\lambda^{H+\varepsilon}-\lambda^{H}-\varepsilon D \lambda^{H}\right\|_{\beta}^{2 \beta} \leq c \varepsilon^{-2 \beta} \int_{0}^{T}\left(\mathbb{E}\left[I_{\varepsilon}^{1}(t)^{2 \beta}\right]+\mathbb{E}\left[I_{\varepsilon}^{2}(t)^{2 \beta}\right]\right) d t .
$$

Estimation for $I_{1}^{\varepsilon}$. By computing the $2 \beta$-moment of the Gaussian random variable $I_{\varepsilon}^{1}$, we obtain

$$
\varepsilon^{-2 \beta} \mathbb{E}\left[I_{\varepsilon}^{1}(t)^{2 \beta}\right] \leq c\left(\int_{\mathbb{R}}\left|\frac{K^{H+\varepsilon}(t, s)-K^{H}(t, s)}{\varepsilon}-\partial_{H} K^{H}(t, s)\right|^{2} d s\right)^{\beta}
$$

which tends to zero by Lemma 7.3 . Furthermore, if $|\varepsilon|<\delta$ for some $\delta>0$, this term is bounded by $c\left(t^{2 H+1}\left(1+(\ln t)^{2}\right)\right)^{\beta}$ if $t \geq 1$ and $c\left(t^{2 H-2 \delta}\left(1+(\ln t)^{2}\right)\right)^{\beta}$ if $0 \leq t<1$. 
Estimation for $I_{\varepsilon}^{2}$. On the one hand, we see that

$$
\begin{aligned}
& \varepsilon^{-2 \beta} \mathbb{E}\left[I_{\varepsilon}^{2}(t)^{2 \beta}\right] \leq \alpha \int_{0}^{t} e^{-2 \alpha \beta(t-u)} \mathbb{E}\left[\left|\frac{B_{u}^{H+\varepsilon}-B_{u}^{H}}{\varepsilon}-\int_{\mathbb{R}} \partial_{H} K^{H}(u, s) d W_{s}\right|^{2 \beta}\right] d u \\
& \leq \alpha\left(\int_{0}^{t} e^{-2 \alpha(t-u)}\left(\int_{\mathbb{R}}\left|\frac{K^{H+\varepsilon}(u, s)-K^{H}(u, s)}{\varepsilon}-\partial_{H} K^{H}(u, s)\right|^{2} d s\right)^{\beta} d u\right)
\end{aligned}
$$

which tends to zero by Lemma 7.3 and the dominated convergence theorem. On the other hand, we obtain

$$
\begin{aligned}
\varepsilon^{-2 \beta} \mathbb{E}\left[I_{\varepsilon}^{2}(t)^{2 \beta}\right] \leq & c \int_{0}^{t \wedge 1} e^{-2 \alpha(t-u)}\left(u^{2 H-2 \delta}\left(1+(\ln u)^{2}\right)\right)^{\beta} d u \\
& +c \int_{1}^{t \vee 1} e^{-2 \alpha(t-u)}\left(u^{2 H+1}\left(1+(\ln u)^{2}\right)\right)^{\beta} d u .
\end{aligned}
$$

Using the dominated convergence theorem again, the convergence (14) holds true. In conclusion, the Fréchet derivative of the process $\lambda^{H}$ is found.

Putting together Theorem 3.4 and Proposition 4.2, we obtain the derivative of the value function $u^{H}(x):=u^{\lambda^{H}}(x)$ with respect to the Hurst parameter $H$.

Theorem 4.3. The mapping $H \mapsto u^{H}(1)$ from $(0,1)$ to $\mathbb{R}$ is differentiable. Its derivative is given by

$$
p u^{\lambda^{H}} \mathbb{E}\left[\frac{d \tilde{\mathbb{P}}^{\lambda^{H}}}{d \mathbb{P}} \int_{0}^{T}\left(D \lambda_{t}^{H}\right) d R_{t}^{\lambda^{H}}\right]
$$

The most interesting consequence of this result is perhaps the case $H=1 / 2$.

Corollary 4.4. At $H=\frac{1}{2}$, we have

$$
u^{\frac{1}{2}+\varepsilon}-u^{\frac{1}{2}}=\varepsilon p u^{\lambda^{\frac{1}{2}}} \mathbb{E}\left[\frac{d \tilde{\mathbb{P}}^{\lambda^{\frac{1}{2}}}}{d \mathbb{P}} \int_{0}^{T}\left(D \lambda_{t}^{\frac{1}{2}}\right) d R_{t}^{\lambda^{\frac{1}{2}}}\right]+o(\varepsilon)
$$

and the first order term could be evaluated numerically.

Proof. First, we compute the derivative of $\lambda^{H}$ at $H=\frac{1}{2}$,

$D \lambda_{t}^{\frac{1}{2}}=C\left(\frac{1}{2}\right) \int_{-\infty}^{0} \ln (1+t / s) d W_{s}+\int_{0}^{t}\left(\partial_{H} C\left(\frac{1}{2}\right)+C\left(\frac{1}{2}\right) \ln (t-s)\right) d W_{s}$.

Second, plugging this derivative into the Gâteaux derivative of $u$ given in Theorem 3.3 gives us the derivative of $u$ at $H=1 / 2$ :

$$
p u^{\lambda^{\frac{1}{2}}} \mathbb{E}\left[\frac{d \tilde{\mathbb{P}}^{\lambda^{\frac{1}{2}}}}{d \mathbb{P}} \int_{0}^{T}\left(D \lambda_{t}^{\frac{1}{2}}\right) d R_{t}^{\lambda^{\frac{1}{2}}}\right]
$$

Recall that, by results of Kim and Omberg (1996), $u^{1 / 2}$ and $\tilde{\mathbb{P}}^{\lambda^{1 / 2}}$ are explicit modulo solving ordinary differential equations. Hence the first order term can indeed be calculated with appropriate Monte Carlo simulations. 
The expansion in Corollary 4.4 gives us an approximation for the value function near $H=\frac{1}{2}$. As the optimization with $H \neq \frac{1}{2}$ is no longer Markovian and an efficient approach for finding its optimizer is unavailable, the above approximations are valuable.

\section{2 $\quad$ Model 2}

In this subsection, we consider the case of memory in log volatility. The following model is suggested in Gatheral et al. (2014)

$$
\begin{aligned}
S_{t}^{H} & =\mathcal{E}\left(R^{H}\right)_{t}, \\
d R_{t}^{H} & =\mu\left(R_{t}^{H}\right) d t+\rho e^{\sigma_{t}^{H}} d W_{t}+\sqrt{1-\rho^{2}} e^{\sigma_{t}^{H}} d B_{t}, \\
d \sigma_{t}^{H} & =-\alpha \sigma_{t}^{H}+d B_{t}^{H} .
\end{aligned}
$$

First, let us define an auxiliary market

$$
\begin{aligned}
\tilde{S}_{t}^{H} & =\mathcal{E}\left(\tilde{R}^{H}\right)_{t}, \\
d \tilde{R}_{t}^{H} & =\frac{\mu\left(R_{t}^{H}\right)}{e^{\sigma_{t}^{H}}} d t+\rho d W_{t}+\sqrt{1-\rho^{2}} d B_{t} .
\end{aligned}
$$

This auxiliary market shifts the problematic factors involving long memory of the $\log$ volatility into the drift, which makes it possible to apply expansion results. To begin with, we will prove that this shifting argument preserve the structure of admissible strategies in the original market.

Lemma 4.5. An strategy $\pi$ is admissible for the $S^{H}$-market if and only if $\tilde{\pi}:=\pi e^{\sigma^{H}}$ is an admissible strategy for the $\tilde{S}^{H}$-market. Furthermore, it holds that $X^{\pi}=X^{\tilde{\pi}}$.

Proof. Since $\pi$ is an admissible strategy in the $S^{H}$-market, one has easily that $\int_{0}^{T} \pi_{t}^{2} e^{2 \sigma_{t}^{H}} d t<+\infty$. This is also the requirement for the admissibility of the strategy $\pi e^{\sigma^{H}}$ in the $\tilde{S}^{H}$-market. A straightforward computation shows that

$$
d X_{t}^{\pi}=\pi_{t} d R_{t}^{H}=\left(\pi e^{\sigma_{t}^{H}}\right)\left(e^{-\sigma_{t}^{H}} d R_{t}^{H}\right)=\tilde{\pi}_{t} d \tilde{R}_{t}^{H}=d X_{t}^{\tilde{\pi}},
$$

and this implies the second conclusion.

As a result, Lemma 4.5 tells us that the solution of the optimization problem in the $S^{H}$-market is also the solution of that problem in the $\tilde{S}^{H}$-market, which can be studied along the lines of Section 4.1. For simplicity, we assume $\mu:=1$.

Proposition 4.6. The mapping $H \mapsto e^{-\sigma_{t}^{H}}$ is Fréchet differentiable and its derivative is the process $-e^{-\sigma_{t}^{H}} D \lambda_{t}^{H}$, where $D \lambda_{t}^{H}$ is exactly as in (13).

Proof. We will prove that $\varepsilon^{-1}\left\|e^{-\sigma^{H+\varepsilon}}-e^{-\sigma^{H}}+\varepsilon e^{-\sigma^{H}} D \lambda^{H}\right\|_{\beta} \rightarrow 0$ as $\varepsilon$ tends to zero, or equivalently,

$$
\mathbb{E}\left[\left(\int_{0}^{T}\left|\frac{e^{-\sigma_{t}^{H+\varepsilon}}-e^{-\sigma_{t}^{H}}}{\varepsilon}+e^{-\sigma_{t}^{H}} D \lambda_{t}^{H}\right|^{2} d t\right)^{\beta}\right] \rightarrow 0 .
$$


Since the quantity $\varepsilon^{-1}\left(e^{-\sigma_{t}^{H+\varepsilon}}-e^{-\sigma_{t}^{H}}\right)-e^{-\sigma_{t}^{H}} D \lambda_{t}^{H}$ convergences to zero in $\mathbb{P} \times$ Leb, it suffices to check its uniform integrability to establish the above convergence. The mean value theorem implies that

$$
\frac{e^{-\sigma_{t}^{H+\varepsilon}+\sigma_{t}^{H}}-1}{\varepsilon}=-e^{\tilde{\varepsilon}\left(-\sigma_{t}^{H+\varepsilon}+\sigma_{t}^{H}\right)} \frac{\sigma_{t}^{H+\varepsilon}-\sigma_{t}^{H}}{\varepsilon}
$$

for some $|\tilde{\varepsilon}|<1$. Therefore, for some $\beta^{\prime}>\beta$, one estimates using Hölder's inequality,

$$
\mathbb{E}\left[\left|\frac{e^{-\sigma_{t}^{H+\varepsilon}-\sigma_{t}^{H}}-1}{\varepsilon}\right|^{2 \beta^{\prime}}\right] \leq \mathbb{E}\left[e^{4 \beta^{\prime} \tilde{\varepsilon}\left(-\sigma_{t}^{H+\varepsilon}+\sigma_{t}^{H}\right)}\right]^{1 / 2} \mathbb{E}\left[\left|\frac{\sigma_{t}^{H+\varepsilon}-\sigma_{t}^{H}}{\varepsilon}\right|^{4 \beta^{\prime}}\right]^{1 / 2}
$$

We notice that $\sigma_{t}^{H+\varepsilon}-\sigma_{t}^{H}$ is a Gaussian random variable with zero mean. An upper bound for its variance is found as follows

$$
\begin{array}{r}
\mathbb{E}\left[\left(\sigma_{t}^{H+\varepsilon}-\sigma_{t}^{H}\right)^{2}\right] \leq 2 \mathbb{E}\left[\left(B_{t}^{H+\varepsilon}-B_{t}^{H}\right)^{2}\right]+2 \mathbb{E}\left[\alpha^{2}\left(\int_{0}^{t} e^{-\alpha(t-u)}\left(B_{u}^{H+\varepsilon}-B_{u}^{H}\right) d u\right)^{2}\right] \\
\leq 2 \mathbb{E}\left[\left(B_{t}^{H+\varepsilon}-B_{t}^{H}\right)^{2}\right]+2 \alpha^{2} \int_{0}^{t} e^{-2 \alpha(t-u)} d u \int_{0}^{t} \mathbb{E}\left[\left(B_{u}^{H+\varepsilon}-B_{u}^{H}\right)^{2}\right] d u .
\end{array}
$$

Using Lemma 7.1] we obtain

$$
\mathbb{E}\left[\left(B_{t}^{H+\varepsilon}-B_{t}^{H}\right)^{2}\right] \leq 2 \int_{\mathbb{R}}\left|K^{H+\varepsilon}(t, s)\right|^{2}+\left|K^{H}(t, s)\right|^{2} d s \leq c\left(t^{2 H+2 \varepsilon}+t^{2 H}\right) .
$$

The variance is bounded by

$$
c\left(t^{2 H+2 \varepsilon}+t^{2 H}+\alpha\left(1-e^{-2 \alpha t}\right)\left(\frac{t^{2 H+2 \varepsilon}}{2 H+2 \varepsilon+1}+\frac{t^{2 H+1}}{2 H+1}\right)\right) .
$$

By using the moment generating function of a normal distribution, we find an upper bound for $\mathbb{E}\left[e^{4 \beta^{\prime} \tilde{\varepsilon}\left(-\sigma_{t}^{H+\varepsilon}+\sigma_{t}^{H}\right)}\right]$ and hence, it holds that

$$
\sup _{|\varepsilon|<\delta} \int_{0}^{T} \mathbb{E}\left[e^{4 \beta^{\prime} \tilde{\varepsilon}\left(-\sigma_{t}^{H+\varepsilon}+\sigma_{t}^{H}\right)}\right] d t<\infty
$$

One can prove, similarly to the argument in the proof of Proposition 4.2, that

$$
\sup _{|\varepsilon|<\delta} \int_{0}^{T} \mathbb{E}\left[\left|\frac{\sigma_{t}^{H+\varepsilon}-\sigma_{t}^{H}}{\varepsilon}\right|^{4 \beta^{\prime}}\right] d t<\infty
$$

The uniform integrability is then satisfied,

$$
\sup _{|\varepsilon|<\delta} \mathbb{E}\left[\int_{0}^{T}\left|\frac{e^{-\sigma_{t}^{H+\varepsilon}}-e^{-\sigma_{t}^{H}}}{\varepsilon}\right|^{2 \beta^{\prime}} d t\right]<\infty,
$$

and thus the proof is complete.

As usual, we define $u^{H}(x):=u^{e^{-\sigma^{H}}}(x)$ for the $\tilde{S}$-market. In Model $2, u^{1 / 2}$ is not known explicitly but it seems possible to calculate it using the corresponding Hamilton-Jacobi-Bellmann equation (recall that $H=1 / 2$ corresponds to a Markovian optimal investment problem). We do not treat such ramifications in the present paper, neither do we pursue the numerical evaluation of (15) above. These are left for future research. 


\section{$5 \quad$ Further applications}

Fix $\mu \in \mathbb{R}$ and consider the process $\lambda^{\varepsilon}:=\mu+\nu^{\varepsilon}$ where

$$
d \nu_{t}^{\varepsilon}=-\frac{1}{\varepsilon} \nu_{t}^{\varepsilon} d t+d W_{t}, \nu_{0}^{\varepsilon}=0
$$

for $\varepsilon>0, W$ is standard Brownian motion and set $\nu_{t}^{0}:=0, \lambda_{t}^{0}:=\mu, t \in[0, T]$.

When $M$ is a Brownian motion (possibly correlated with $W$ ), this choice of drift corresponds to continuous-time FADS models, see Guasoni (2006), where mean-reversion is intensifying to infinity as $\varepsilon \rightarrow 0$ (thus the limit is the constant drift $\mu$ ).

Results of Section 3 enable us to get an asymptotic result on the value of $u^{\varepsilon}(x):=u^{\lambda^{\varepsilon}}(x)$ when $\varepsilon \rightarrow 0$, i.e. when mean reversion is strong.

Proposition 5.1. Let $M$ be such that $d\langle M\rangle_{t} \leq G d t$ with some constant $G>0$. Then, for each $0<\delta<1 / 2$,

$$
u^{\varepsilon}-u^{0}=o\left(\varepsilon^{\delta}\right)
$$

Remark 5.2. When $M=\rho W+\sqrt{1-\rho^{2}} B$ with a Brownian motion $B$ independent of $W$ and the filtration is the one generated by $(W, B)$ then the optimal utilities $u^{\varepsilon}$ can be explicitly calculated, see Kim and Omberg (1996) and Battauz et al. (2015). Proposition 5.1 derives the asymptotics of the value functions for other choices of $M$ as well, where no explicit solution can be expected. We cannot determine the first order expansion but we can still estimate the magnitude of the deviation of the optimal utility $u^{\varepsilon}$ from that of the benchmark model, $u^{0}$.

Proof of Proposition 5.1. Let us consider, instead of $\nu^{\varepsilon}$,

$$
d \nu_{t}^{\eta}=-\frac{1}{\eta^{\kappa}} \nu_{t}^{\eta} d t+d W_{t}, \nu_{0}^{\eta}=0
$$

for some $\kappa>2$, setting $\lambda^{\eta}:=\nu^{\eta}+\mu$ and $\nu^{0}:=0, \lambda^{0}:=\mu$. The explicit formula for $\nu^{\eta}$ is

$$
\nu_{t}^{\eta}=e^{-t / \eta^{\kappa}} \int_{0}^{t} e^{s / \eta^{\kappa}} d W_{s} .
$$

We prove that the (Fréchet) right-hand derivative of $\eta \rightarrow \nu^{\eta} \in \mathcal{D}_{\beta}$ is 0 , i.e.

$\left\|\frac{\nu^{\eta}-\nu^{0}}{\eta}\right\|_{\beta}^{2 \beta}=\mathbb{E}\left[\left(\int_{0}^{T} \frac{\left(\nu_{t}^{\eta}\right)^{2}}{\eta^{2}} d\langle M\rangle_{t}\right)^{\beta}\right] \leq G^{\beta} \mathbb{E}\left[\left(\int_{0}^{T} \frac{\left(\nu_{t}^{\eta}\right)^{2}}{\eta^{2}} d t\right)^{\beta}\right] \rightarrow 0, \eta \rightarrow 0$.

In the sequel $C>0$ denotes a constant whose value may change from line to 
line. Denoting by $N^{\eta}$ the martingale $\int_{0}^{\cdot} e^{s / \eta^{\kappa}} d W_{s}$, we have

$$
\begin{aligned}
\mathbb{E}\left[\left(\int_{0}^{T}\left(\nu_{t}^{\eta}\right)^{2} d t\right)^{\beta}\right] & =\mathbb{E}\left[\left(\int_{0}^{T} e^{-2 t / \eta^{\kappa}}\left(N_{t}^{\eta}\right)^{2} d t\right)^{\beta}\right] \\
& \leq \mathbb{E}\left[\left(\int_{0}^{T} e^{-2 t / \eta^{\kappa}} \sup _{s \in[0, t]}\left|N_{s}^{\eta}\right|^{2} d t\right)^{\beta}\right] \\
& \leq C \mathbb{E}\left[\int_{0}^{T} e^{-2 \beta t / \eta^{\kappa}} \sup _{s \in[0, t]}\left|N_{s}^{\eta}\right|^{2 \beta} d t\right] \\
& \leq C \int_{0}^{T} e^{-2 \beta t / \eta^{\kappa}} \mathbb{E}\left[\sup _{s \in[0, t]}\left|N_{s}^{\eta}\right|^{2 \beta}\right] d t .
\end{aligned}
$$

The Burkholder-Davis-Gundy inequality shows that

$$
\mathbb{E}\left[\sup _{s \in[0, t]}\left|N_{s}^{\eta}\right|^{2 \beta}\right] \leq C\left(\int_{0}^{t} e^{2 s / \eta^{\kappa}} d s\right)^{\beta}=C\left(\frac{e^{2 t / \eta^{\kappa}}-1}{2 / \eta^{\kappa}}\right)^{\beta}
$$

hence

$$
\mathbb{E}\left[\left(\int_{0}^{T}\left(\nu_{t}^{\eta}\right)^{2} / \eta^{2} d t\right)^{\beta}\right] \leq \frac{C}{\eta^{2 \beta}} \int_{0}^{T} e^{-2 \beta t / \eta^{\kappa}}\left(\frac{e^{2 t / \eta^{\kappa}}-1}{2 / \eta^{\kappa}}\right)^{\beta} d t \rightarrow 0,
$$

as $\eta$ tends to zero. This clearly entails that the derivative of $\eta \rightarrow \lambda^{\eta}$ is also 0 . Noting the Fréchet differentiability of $\lambda \rightarrow u^{\lambda}$ we get that $u^{\eta}-u^{0}=o(\eta)$ where $u^{\eta}$ is the optimal utility for the drift process $\nu^{\eta}$. Now apply this with $\kappa:=1 / \delta$ and $\varepsilon:=\eta^{\kappa}$ to conclude that (16) indeed holds true.

\section{Conclusions}

Here we discuss certain important ramifications that deserve to be addressed in more detail. A relevant question is whether one may get an approximation of the optimal strategy $\pi^{\lambda}$ instead of the value function $u^{\lambda}$. As we have already pointed out in Remark 3.6 above, Subsection 3.2 of Larsen et al. (2016) mentions such a result.

The arguments of Larsen et al. (2016) work along a specific directional derivative, i.e. they study $\pi^{\lambda+\varepsilon \lambda^{\prime}}$ with fixed $\lambda^{\prime}$ and require technical assumptions that are not easy to check (they are in terms of $\tilde{\mathbb{P}}^{\lambda}$ about which we have little information in general), see Theorem 3.4 of Larsen et al. (2016). A natural continuation of our present work would be to strengthen their arguments so as to yield the "almost optimality" of (9) uniformly along all directions $\lambda^{\prime}$ and preferably under sufficient conditions that are easy to check.

We shortly explain in a one-period model of a financial market what such an extension should involve. Let us try to maximize

$$
\mathbb{E}\left[U\left(\phi R^{\lambda}\right)\right]
$$


where, for convenience, we assume that the concave and continuously differentiable utility function $U$ is defined and finite on $\mathbb{R}, \phi \in \mathbb{R}$ is the number of units of the stock that is bought (this represents the portfolio strategy), and the random variable $R^{\lambda}$ is the return on the given stock, parametrized by some $\lambda \in \mathbb{R}$. (We assume that the agent has 0 initial capital.)

Under appropriate conditions, the optimal strategy $\phi(\lambda)$ satisfies

$$
\mathbb{E}\left[U^{\prime}\left(\phi(\lambda) R^{\lambda}\right) R^{\lambda}\right]=0 .
$$

If the implicit function theorem is applicable then $\lambda \rightarrow \phi(\lambda)$ is continuously differentiable and its derivative satisfies

$$
\begin{aligned}
\partial_{\lambda} \phi(\lambda) \mathbb{E}\left[U^{\prime \prime}\left(\phi(\lambda) R^{\lambda}\right)\left(R^{\lambda}\right)^{2}\right] & +\mathbb{E}\left[U^{\prime \prime}\left(\phi(\lambda) R^{\lambda}\right) \phi(\lambda) R^{\lambda} \partial_{\lambda} R^{\lambda}\right] \\
& +\mathbb{E}\left[U^{\prime}\left(\phi(\lambda) R^{\lambda}\right) \partial_{\lambda} R^{\lambda}\right]=0 .
\end{aligned}
$$

From this we can express $\partial_{\lambda} \phi(\lambda)$ and obtain the first-order correction term of $\lambda \rightarrow \phi(\lambda)$ for $\lambda$ in a neighbourhood of some fixed $\lambda^{*}$, i.e. $\phi(\lambda) \approx \phi\left(\lambda^{*}\right)+(\lambda-$ $\left.\lambda^{*}\right) \partial_{\lambda} \phi\left(\lambda^{*}\right)$. Higher order correction terms can be deduced in an analogous way.

It is quite clear that transferring the above arguments to the continuoustime setting looks highly non-trivial: what kind of infinite-dimensional Banach spaces should be used for the strategy and for the (drift) parameter so that a similar argument can go through? From Subsection 3.2 of Larsen et al. (2016) we do know the first-order correction term (though it is not explicit enough due to the presence of $\gamma^{B}$, see (9) above) hence it would remain to establish that the obtained representations hold uniformly in all directions.

An extension of the optimal strategy in the model space thus requires further research effort which has started but certainly leads beyond the scope of the present paper.

\section{Appendix}

In this section, we provide some useful results for fractional Brownian motion.

Lemma 7.1. The functions $C_{1}(\alpha), C_{2}(\alpha)$ are continuous on $\left(-\frac{1}{2}, \frac{1}{2}\right)$. In addition, we have that

$$
\begin{gathered}
\int_{\mathbb{R}}\left((t-s)_{+}^{\alpha}-(-s)_{+}^{\alpha}\right)^{2} d s=t^{2 \alpha+1} C_{1}(\alpha), \\
\int_{\mathbb{R}}\left((t-s)_{+}^{\alpha} \ln (t-s)_{+}-(-s)_{+}^{\alpha} \ln (-s)_{+}\right)^{2} d s \leq 2 t^{2 \alpha+1}(\ln t)^{2} C_{1}(\alpha)+2 t^{2 \alpha+1} C_{2}(\alpha) .
\end{gathered}
$$

Proof. The continuity of $C_{1}$ comes from the continuity of $C$. We now consider the function $C_{2}$. Letting $\varepsilon \rightarrow 0$, it holds that

$$
\left|(1+s)^{\alpha+\varepsilon} \ln (1+s)-s^{\alpha+\varepsilon} \ln s\right| \rightarrow\left|(1+s)^{\alpha} \ln (1+s)-s^{\alpha} \ln s\right| .
$$

Fix $\delta<\alpha$. We separate two cases.

The case $\alpha \in(0,1 / 2)$. For $|\varepsilon|<\delta$ then

$$
\left|(1+s)^{\alpha+\varepsilon} \ln (1+s)-s^{\alpha+\varepsilon} \ln s\right| \leq\left|(1+s)^{\alpha+\delta} \ln (1+s)-s^{\alpha+\delta} \ln s\right|, \text { if } s>1 .
$$


If $0<s \leq 1$, since $s^{\alpha+\varepsilon} \ln s \rightarrow 0$ as $s$ tends to zero, we can find a constant such that $\left|(1+s)^{\alpha+\varepsilon} \ln (1+s)-s^{\alpha+\varepsilon} \ln s\right| \leq c$. Thus, the dominated convergence theorem implies $C_{2}(\alpha+\varepsilon) \rightarrow C_{2}(\alpha)$.

The case $\alpha \in(-1 / 2,0)$. Let $|\varepsilon|<\delta$, there exists $K$ big enough such that $\left|(1+s)^{\alpha+\varepsilon} \ln (1+s)-s^{\alpha+\varepsilon} \ln s\right| \leq\left|(1+s)^{\alpha-\delta} \ln (1+s)-s^{\alpha-\delta} \ln s\right|$, if $s>K$.

If $s<K$, we estimate $\left|(1+s)^{\alpha+\varepsilon} \ln (1+s)-s^{\alpha+\varepsilon} \ln s\right| \leq m+s^{\alpha+\varepsilon} \ln s$ for some $m>0$. Furthermore, we compute

$$
\begin{aligned}
\int_{0}^{K}\left(s^{\alpha+\varepsilon} \ln s\right)^{2} d s & =\left.\frac{s^{2(\alpha+\varepsilon)+1}(\ln s)^{2}}{2(\alpha+\varepsilon)+1}\right|_{0} ^{K} \\
& -\left.\frac{1}{2(\alpha+\varepsilon)+1} s^{2(\alpha+\varepsilon)+1}\left(\frac{\ln s}{2(\alpha+\varepsilon)+1}-\frac{1}{(2(\alpha+\varepsilon)+1)^{2}}\right)\right|_{0} ^{K} .
\end{aligned}
$$

Now, applying the extended dominated convergence theorem to the sequence $\left|(1+s)^{\alpha+\varepsilon} \ln (1+s)-s^{\alpha+\varepsilon} \ln s\right|^{2}$ which is smaller than

$$
1_{s>K}\left|(1+s)^{\alpha-\delta} \ln (1+s)-s^{\alpha-\delta} \ln s\right|^{2}+1_{s \leq K}\left(m^{2}+s^{2(\alpha+\varepsilon)} \ln ^{2} s\right),
$$

we obtain $C_{2}(\alpha+\varepsilon) \rightarrow C_{2}(\alpha)$.

Let us consider the first equality. By changing of variable $s=t x$, one has

$$
\begin{aligned}
\int_{\mathbb{R}}\left((t-s)_{+}^{\alpha}-(-s)_{+}^{\alpha}\right)^{2} d s & =\int_{0}^{\infty}\left((t+s)^{\alpha}-s^{\alpha}\right)^{2} d s+\int_{0}^{t}(t-s)^{2 \alpha} d s \\
& =t^{2 \alpha+1}\left(\int_{0}^{\infty}\left((1+x)^{\alpha}-(x)^{\alpha}\right)^{2} d s+\int_{0}^{1}(1-x)^{2 \alpha} d x\right)
\end{aligned}
$$

and the first identity follows. Now, straightforward computations show that $(t+s)^{\alpha} \ln (t+s)-s^{\alpha} \ln s=t^{\alpha} \ln t\left[(1+x)^{\alpha}-x^{\alpha}\right]+t^{\alpha}\left[(1+x)^{\alpha} \ln (1+x)-x^{\alpha} \ln x\right]$ and $(t-s)^{\alpha} \ln (t-s)=t^{\alpha} \ln t(1-x)^{\alpha}+t^{\alpha}(1-x)^{\alpha} \ln (1-x)$. Therefore,

$$
\begin{aligned}
& \int_{\mathbb{R}}\left((t-s)_{+}^{\alpha} \ln (t-s)_{+}-(-s)_{+}^{\alpha} \ln (-s)_{+}\right)^{2} d s=\int_{0}^{t}\left((t-s)^{\alpha} \ln (t-s)\right)^{2} d s \\
& +\int_{0}^{\infty}\left((t+s)^{\alpha} \ln (t+s)-s^{\alpha} \ln s\right)^{2} d s \leq 2 t^{2 \alpha+1}(\ln t)^{2} \int_{0}^{1}(1-x)^{2 \alpha} d x \\
& +2 t^{2 \alpha+1} \int_{0}^{1}\left[(1-x)^{\alpha} \ln (1-x)\right]^{2} d x+2 t^{2 \alpha+1}(\ln t)^{2} \int_{0}^{\infty}\left((1+x)^{\alpha}-x^{\alpha}\right)^{2} d x \\
& +2 t^{2 \alpha+1} \int_{0}^{\infty}\left((1+x)^{\alpha} \ln (1+x)-x^{\alpha} \ln x\right)^{2} d x .
\end{aligned}
$$

From that, the proof is complete.

Lemma 7.2. The derivative of the function $K^{H}$ is

$$
\begin{aligned}
\partial_{H} K^{H}(t, s) & =\partial_{H} C(H)\left((t-s)_{+}^{H-\frac{1}{2}}-(-s)_{+}^{H-\frac{1}{2}}\right) \\
& +C(H)\left((t-s)_{+}^{H-\frac{1}{2}} \ln (t-s)_{+}-(-s)_{+}^{H-\frac{1}{2}} \ln (-s)_{+}\right) .
\end{aligned}
$$

Furthermore, the derivative is square integrable over $\mathbb{R}$. 
Proof. Since the function $C(H)$ is smooth in $H$, we get the derivative of $K^{H}$ with respect to $H$. The second statement is deduced from Lemma 7.1.

Lemma 7.3. As $\varepsilon$ goes to zero, the following convergence holds, for any $t \geq 0$,

$$
\int_{\mathbb{R}}\left|\frac{K^{H+\varepsilon}(t, s)-K^{H}(t, s)}{\varepsilon}-\partial_{H} K^{H}(t, s)\right|^{2} d s \rightarrow 0 .
$$

In addition, the integral is bounded by

$$
\begin{cases}c t^{2 H+1}\left(1+(\ln t)^{2}\right) & \text { if } t \geq 1 \\ c t^{2 H-2 \delta}\left(1+(\ln t)^{2}\right) & \text { if } 0 \leq t<1,|\varepsilon|<\delta<H,\end{cases}
$$

which is a function in $L^{\beta}(0, T)$ for $\beta>1$.

Proof. First, we notice that

$$
\lim _{\varepsilon \rightarrow 0}\left[\frac{K^{H+\varepsilon}(t, s)-K^{H}(t, s)}{\varepsilon}-\partial_{H} K^{H}(t, s)\right]=0, \quad \text { for each } t, s .
$$

By the mean value theorem, $K^{H+\varepsilon}(t, s)-K^{H}(t, s)=\varepsilon \partial_{H} K^{H+\tilde{\varepsilon}}(t, s)$ for some $\tilde{\varepsilon}=\tilde{\varepsilon}(t, s)$. Thus, it is immediate that

$$
\left|\frac{K^{H+\varepsilon}(t, s)-K^{H}(t, s)}{\varepsilon}-\partial_{H} K^{H}(t, s)\right|^{2} \leq 2\left(\left|\partial_{H} K^{H+\tilde{\varepsilon}}(t, s)\right|^{2}+\left|\partial_{H} K^{H}(t, s)\right|^{2}\right) \text {. }
$$

Since $\partial_{H} K^{H+\tilde{\varepsilon}}(t, s) \rightarrow \partial_{H} K^{H}(t, s)$ for all $t, s$ when $\varepsilon \rightarrow 0$ and

$$
\lim _{\varepsilon \rightarrow 0} \int_{\mathbb{R}} 2\left(\left|\partial_{H} K^{H+\tilde{\varepsilon}}(t, s)\right|^{2}+\left|\partial_{H} K^{H}(t, s)\right|^{2}\right) d s=4 \int_{\mathbb{R}}\left|\partial_{H} K^{H}(t, s)\right|^{2} d s
$$

by the formula (18) and Lemma 7.1, an application of the extended dominated convergence theorem implies that (19) holds true. We also estimate

$$
\begin{aligned}
& \int_{\mathbb{R}}\left|\partial_{H} K^{H+\tilde{\varepsilon}}(t, s)\right|^{2}+\left|\partial_{H} K^{H}(t, s)\right|^{2} d s \leq 2\left(\partial_{H} C(H+\tilde{\varepsilon})\right)^{2} C_{1}(H-1 / 2+\tilde{\varepsilon}) t^{2 H+2 \tilde{\varepsilon}} \\
& +4 C^{2}(H+\tilde{\varepsilon})\left[C_{1}(H-1 / 2+\tilde{\varepsilon}) t^{2 H+2 \tilde{\varepsilon}}(\ln t)^{2}+C_{2}(H-1 / 2+\tilde{\varepsilon}) t^{2 H+2 \tilde{\varepsilon}}\right] \\
& +2\left(\partial_{H} C(H)\right)^{2} C_{1}(H-1 / 2) t^{2 H}+4 C^{2}(H)\left[C_{1}(H-1 / 2) t^{2 H}(\ln t)^{2}+C_{2}(H-1 / 2) t^{2 H}\right] .
\end{aligned}
$$

Since the functions $C(\cdot), \partial_{H} C(\cdot), C_{1}(\cdot), C_{2}(\cdot)$ are continuous with respect to $\alpha$, there exists $\delta>0$ small enough, such that for all $\varepsilon \in(-\delta, \delta)$, one has

$$
\int_{\mathbb{R}}\left|\frac{K^{H+\varepsilon}(t, s)-K^{H}(t, s)}{\varepsilon}-\partial_{H} K^{H}(t, s)\right|^{2} d s \leq \begin{cases}c t^{2 H+1}\left(1+(\ln t)^{2}\right) & \text { if } t \geq 1 \\ c t^{2 H-2 \delta}\left(1+(\ln t)^{2}\right) & \text { if } 0 \leq t<1 .\end{cases}
$$

with some constant $c=c(H, \delta)>0$.

Theorem 7.4 (The extended dominated convergence theorem). Let $(\Omega, \mathcal{F}, \mu)$ be a measure space and let $f_{n}, g_{n}: \Omega \rightarrow \mathbb{R}$ be measurable functions such that $\left|f_{n}\right| \leq g_{n}$, a.e. for all $n \geq 1$. Suppose that 
- $g_{n} \rightarrow g$, a.e and $f_{n} \rightarrow f$, a.e.

- $g_{n}, g \in L^{1}(\Omega)$ and $\int\left|g_{n}\right| d \mu \rightarrow \int|g| d \mu$ as $n \rightarrow \infty$.

Then $f \in L^{1}(\Omega)$,

$$
\lim _{n \rightarrow \infty} \int f_{n} d \mu=\int f d \mu, \quad \text { and } \quad \lim _{n \rightarrow \infty} \int\left|f_{n}-f\right| d \mu=0 .
$$

Proof. See Theorem 2.3.11 of Athreya and Lahiri (2006).

\section{References}

Athreya, K. B. and Lahiri, S. N. (2006). Measure theory and probability theory. Springer Science \& Business Media.

Battauz, A., De Donno, M., and Sbuelz, A. (2015). Kim and Omberg revisited: the duality approach. Journal of Probability and Statistics, 2015.

Chau, H. N., Cosso, A., Fontana, C., and Mostovyi, O. (2015). Optimal investment with intermediate consumption under no unbounded profit with bounded risk. arXiv:1509.01672.

Cheridito, P., Kawaguchi, H., Maejima, M., et al. (2003). Fractional OrnsteinUhlenbeck processes. Electron. J. Probab, 8(3):1-14.

Comte, F. and Renault, E. (1998). Long memory in continuous-time stochastic volatility models. Mathematical Finance, 8(4):291-323.

Delbaen, F. and Schachermayer, W. (2006). The mathematics of arbitrage. Springer.

Drábek, P. and Milota, J. (2013). Methods of nonlinear analysis: applications to differential equations. Springer Science \& Business Media, 2nd edition.

Fukasawa, M. (2011). Asymptotic analysis for stochastic volatility: martingale expansion. Finance and Stochastics, 15(4):635-654.

Gatheral, J., Jaisson, T., and Rosenbaum, M. (2014). Volatility is rough. Available at SSRN $250945 \%$.

Gerhold, S., Guasoni, P., Muhle-Karbe, J., and Schachermayer, W. (2014). Transaction costs, trading volume, and the liquidity premium. Finance Stoch., 18(1):1-37.

Gerhold, S., Muhle-Karbe, J., and Schachermayer, W. (2012). Asymptotics and duality for the Davis and Norman problem. Stochastics, 84(5-6):625-641.

Gerhold, S., Muhle-Karbe, J., and Schachermayer, W. (2013). The dual optimizer for the growth-optimal portfolio under transaction costs. Finance Stoch., 17(2):325-354.

Giraitis, L., Koul, H. L., and Surgailis, D. (2012). Large sample inference for long memory processes. Imperial College Press. 
Guasoni, P. (2006). Asymmetric information in fads models. Finance and Stochastics, 10(2):159-177.

Guasoni, P. and Weber, M. (2015). Dynamic trading volume. Published online by Math. Finance.

Karatzas, I. and Kardaras, C. (2007). The numéraire portfolio in semimartingale financial models. Finance and Stochastics, 11(4):447-493.

Kim, T. S. and Omberg, E. (1996). Dynamic nonmyopic portfolio behavior. Review of Financial Studies, 9(1):141-161.

Larsen, K., Mostovyi, O., and Žitković, G. (2016). An expansion in the model space in the context of utility maximization. arXiv:1410.0946v3.

Larsen, K. and Žitković, G. (2007). Stability of utility-maximization in incomplete markets. Stochastic Processes and their Applications, 117(11):16421662 .

Willinger, W., Taqqu, M. S., and Teverovsky, V. (1999). Stock market prices and long-range dependence. Finance and Stochastics, 3(1):1-13. 